\title{
Regrasp Planning for Pivoting Manipulation by a Humanoid Robot
}

\author{
Eiichi Yoshida, Mathieu Poirier, Jean-Paul Laumond, Oussama Kanoun, Florent Lamiraux, \\ Rachid Alami and Kazuhito Yokoi.
}

\begin{abstract}
A method of regrasp planning for humanoid robot manipulation is proposed. We adopt pivoting manipulation for the humanoid robot to move a bulky object without lifting in a stable and dexterous manner. In order to carry the object to a desired place, the humanoid should sometimes move through narrow areas surrounded by obstacles. We propose a roadmap multiplexing planning to allow the robot to leave the object near narrow places and to regrasp it from another position to continue carrying. We utilize visibility probabilistic roadmap (PRM) method as a preprocessing to capture the critical configurations for regrasping. Then a diffusion method is employed to plan the overall manipulation path including regrasping. The proposed method is verified through planning simulation including whole-body motions.
\end{abstract}

\section{INTRODUCTION}

Manipulation requiring whole-body motion is one of the tasks that are appropriate for humanoid robots. Currently whole-body humanoid tasks are actively being studied: for instance, pushing [1], [2] or lifting [3], [4]. We have been studying on pivoting [5] that consists of manipulating a bulky object by inclining and rotating. It is advantageous for the humanoid robot in terms of dexterity, stability and security since the robot does not need to lift the object [6]. We have proposed collision-free whole-body motion planning for pivoting in our previous work [7], [8]. Fig. 1 shows an example of whole-body manipulation including coordinated arm and leg motions also by keeping dynamic balance. The manipulated object is carried to the goal position several meters away along the planned curve.

However, the planner proposed in [8] does not allow the robot to change the grasping point: it may be blocked if there are places where the robot and the object cannot pass through together. When we humans transport a bulky object in a cluttered environment, we sometimes release the object and hold it with another position according to the situation.

In this work, we provide a humanoid robot with more flexibility in whole-body pivoting manipulation by including regrasp planning. The robot releases the object when it cannot go further towards the goal position and grasp it again to continue manipulation.

The difficulty resides in finding narrow passages for the robot and object together and in combining the paths with

This work has been done as a joint research in AIST/IS-CNRS/ST2I Joint Japanese-French Robotics Laboratory (JRL).

Eiichi Yoshida and Kazuhito Yokoi are with JRL, Research Institute of Intelligent Systems, National Institute of Advanced Industrial Science and Technology (AIST), 1-1-1 Umezono, Tsukuba, Ibaraki 305-8568 Japan (e.yoshidadaist.go.jp)

Mathieu Poirier, Jean-Paul Laumond, Oussama Kanoun, Florent Lamiraux and Rachid Alami are with LAAS-CNRS, 7 avenue du Colonel Roche, F-31077 Toulouse, and Universite de Toulouse ; UPS, INSA, INP, ISAE ; LAAS ; F-31077 Toulouse, France.
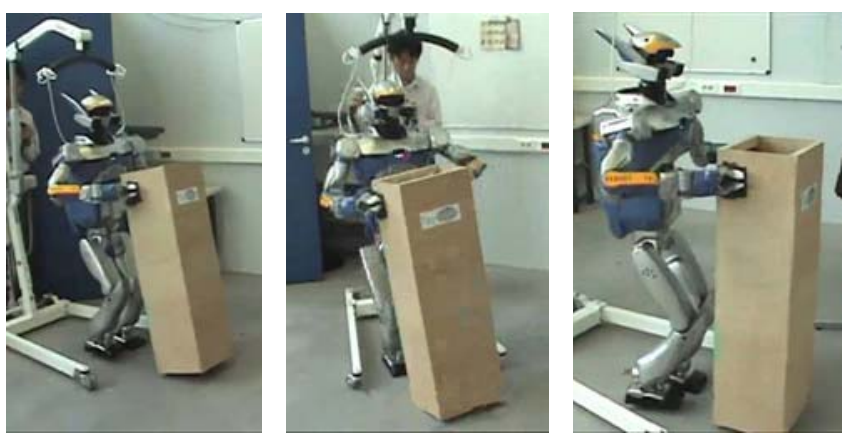

Fig. 1. An experiment of whole-body pivoting manipulation.

different grasping positions to plan a manipulation motion to achieve the goal.

Movable objects have been dealt with using classical motion planners [9], [10], [11] before the introduction of randomized motion planners where different configuration spaces are addressed depending on the relative position of the robot to the movable objects. Simeon et al. [12] proposed a theoretical basis of manipulation planning including "transit" path without the object and "transfer" with it, which allows the manipulation plan with regrasping. In the humanoid related research, Stilman et al. [13], [14] addressed "navigation among movable objects" and Okada et al. [15] proposed an "environment planner" respectively, to allow the humanoid robot to navigate by displacing movable obstacles. Both methods focus on navigation based on search in the graph that represents the structure of the environment.

We here address the regrasp planning problem for pivoting manipulation through a roadmap-multiplexing approach. First, we plan an object-only roadmap to identify "critical configurations" by capturing the global view of the environment based on visibility roadmap planning method [16]. From the obtained object visibility roadmap, multiple "manipulation roadmaps" are constructed for the combined robot-object system for different grasping positions. Those roadmaps are interconnected by a "regrasping roadmap" for free robot walking. A collision-free manipulation path including regrasping is searched by applying randomized diffusion planning method to the whole set of those roadmaps. Finally the whole-body motions are generated based on the method proposed in [8].

The contribution of this work is reinforcement of the flexibility for humanoid manipulation by allowing regrasping and also the integration of visibility roadmap, diffusion planning and whole-body motion generation of humanoid motion. 


\section{Pivoting Manipulation by Humanoid robot}

So far we have been investigating the whole-body manipulation of a polyhedral object by a humanoid robot [6], [7], [8]. It is useful when the object is bulky or heavy since the robot does not lift the object but manipulates it by using the contact support from the floor. We can also expect a dexterous manipulation by using point contacts of the object vertices. As shown in Fig. 2, the 3D pivoting motion of the object is modeled by a 2D segment of which two endpoints are used as a center of horizontal rotation. We have shown a property that any collision-free path is transformed into pivoting sequence based on small-time controllability [7].

The pivoting sequence planning proposed in [8] takes two steps. A supporting collision-free path for the edge is first planned. While manipulating, it is easier for the humanoid to move in the forward direction than sidestepping. By taking this in account as nonholonomic constraints [17], we applied a local steering method called Reeds and Shepp curves [18], composed of line segment and arc of circles allowing backward motions. This steering method enables the robot to go backwards to manipulation the object near a wall.

Once a basic collision-free path is planned, it is transformed into a pivoting sequence of the object. Finally, the whole-body humanoid motion is generated to achieve the hand trajectory that achieves the planned sequence.

With those results at hand, we are now ready to investigate roadmap multiplexing to handle more complex situations.

\section{IDENTIFYING CRITICAL CONFIGURATIONS}

We employ the "visibility" Probabilistic Roadmap (PRM) [16], [19] to help the planner identify the critical configurations. Two types of nodes are considered in visibility roadmap.

1) Guards: To become a guard, a node in the roadmap $q$ must not be able to see other guards. Thus, the visibility region, $V(q)$, must be empty of other guards.

2) Connectors: To become a connector, a node $q$, must see at least two guards. Thus, there exist guards $q_{1}$ and $q_{2}$, such that $q \in V\left(q_{1}\right) \cap V\left(q_{2}\right)$.

Fig. 3 shows the way it works. The black circles are the guards already included in the roadmap $\mathcal{G}$. There are three

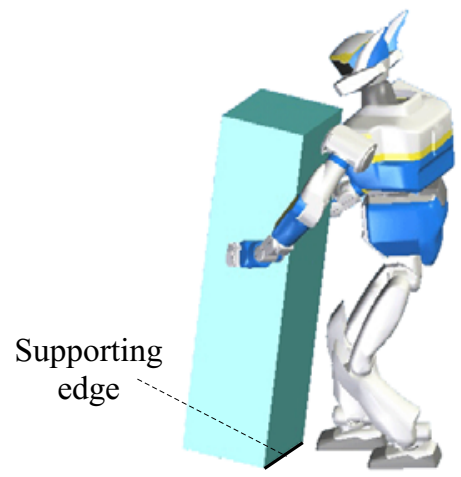

Fig. 2. Supporting edge. The pivoting sequence is planned using rotation of the endpoints of this edge.

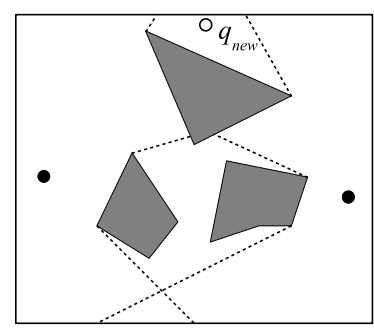

(a)

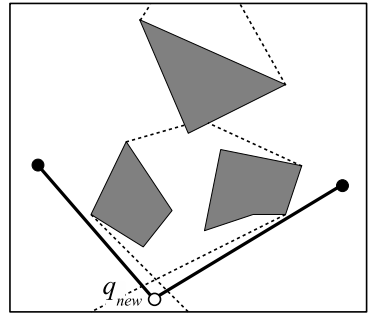

(b)

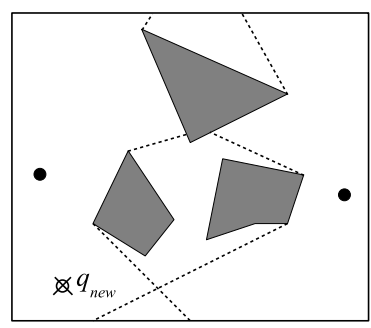

(c)

Fig. 3. Three cases during building the Visibility PRM.

cases when a new configuration $q_{\text {new }}$ is sampled. First, if $q_{\text {new }}$ cannot be connected to any guards, $q_{\text {new }}$ becomes a guard and is inserted into the roadmap (Fig. 3a). The second case is that $q_{\text {new }}$ can be connected to at least two different connected components of $\mathcal{G}$. In this case, $q_{\text {new }}$ becomes a connector that adds associated edges in $\mathcal{G}$ to connect the different connected components (Fig. 3b). If $q_{\text {new }}$ does not satisfy any of those two conditions, it is discarded (Fig. 3c).

The visibility PRM has characteristics to keep the roadmap compact. Since it attempts to connect separate connected components, it helps identifying narrow passages more efficiently than plain PRM.

For those reasons, we adopt this Visibility PRM to capture the global view of the environment. When the humanoid robot carries the object by pivoting manipulation in an environment with obstacles, it may often be blocked by narrow spaces where it cannot go through by holding the object. The Visibility PRM serves as a preprocessing procedure that helps the planner identify possible regrasping points of manipulation.

This preprocessing is illustrated in Fig. 4. First, the visibility PRM is executed with the carried object alone to capture the passages where the object can pass through. Suppose that a new node $q_{\text {new }}^{o b j}$ that is judged to be added to the "object roadmap" $\mathcal{G}_{o b j}$ (Fig. 4a). Before the addition, collisions are checked by attaching the approximated robot bounding volume along the new edges $e_{\text {new }}^{o b j}$ to be added to connect to other connected components. If $e_{n e w}^{o b j}$ is collisionfree, insert it in $\mathcal{G}_{o b j}$.

On the other hand, if $e_{n e w}^{o b j}$ induces collisions between the robot and the obstacles, the collision-free portions $e_{\text {free }}^{r o b}$ are extracted (Fig. 4b). Then $e_{\text {free }}^{r o b}$ are added in $\mathcal{G}_{\text {obj }}$ instead of $e_{\text {new }}^{o b j}$.

The newly inserted nodes $q_{f r e e}^{r o b}$ are considered to represent critical configurations that are the candidates of the regrasping points. Fig. 5 shows an example of object roadmap. We can observe that the narrow areas are roughly covered by the 


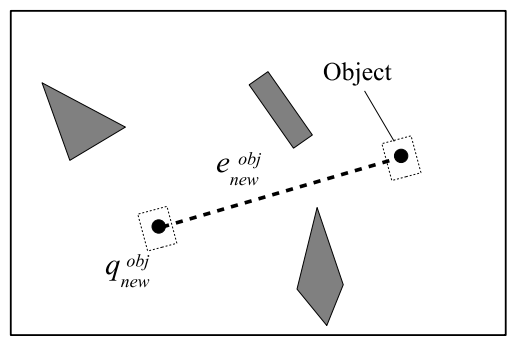

(a)

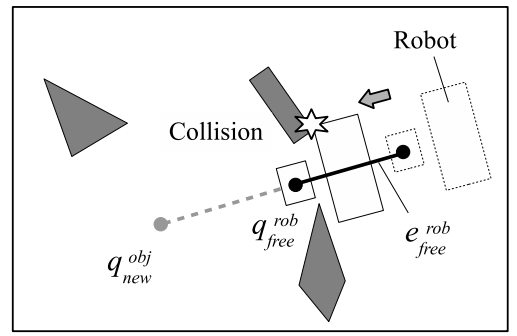

(b)

Fig. 4. Preprocessing of Visibility roadmap for object to identify critical configurations with the robot.

roadmap. Note that the links between the nodes are symbolic to represent the connectivity and their actual motions are subject to nonholonomic constraints of Reeds and Shepp curves for car-like robots. Notice also that the edges between the nodes do not have much importance because we will use only the nodes in construction of roadmap for the combined robot-object system in the next section.

\section{REGRASP PLANNING BY MULTIPLEXING ROADMAPS}

In this section we describe a method of regrasp planning using roadmap multiplexing to combine different grasping positions. Fig. 6 illustrates the overview of the planning scheme. Several grasping positions are possible for a given object position. The roadmaps are built for the combined bounding volumes of both the robot and the object. There

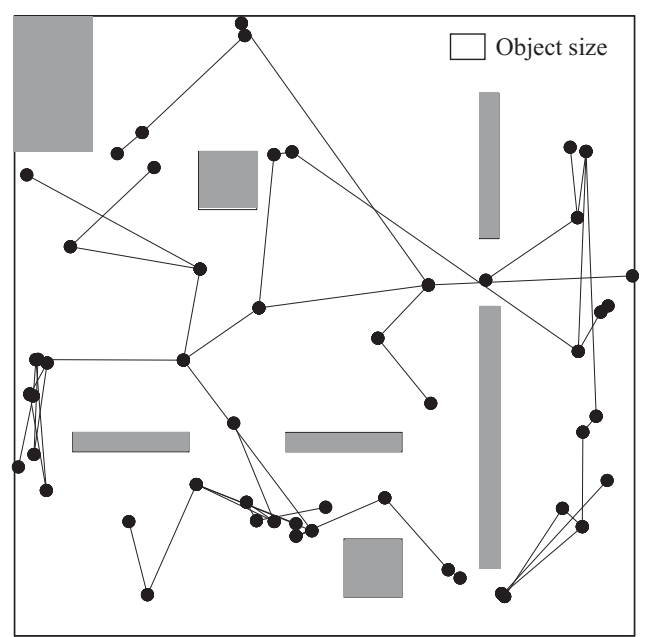

Fig. 5. An example of resulting object roadmap. The link between nodes represents symbolic connections by nonholonomic local steering method. The absence of connection between two nodes apparently close means that the shortest Reeds and Shepp path is not collision-free.

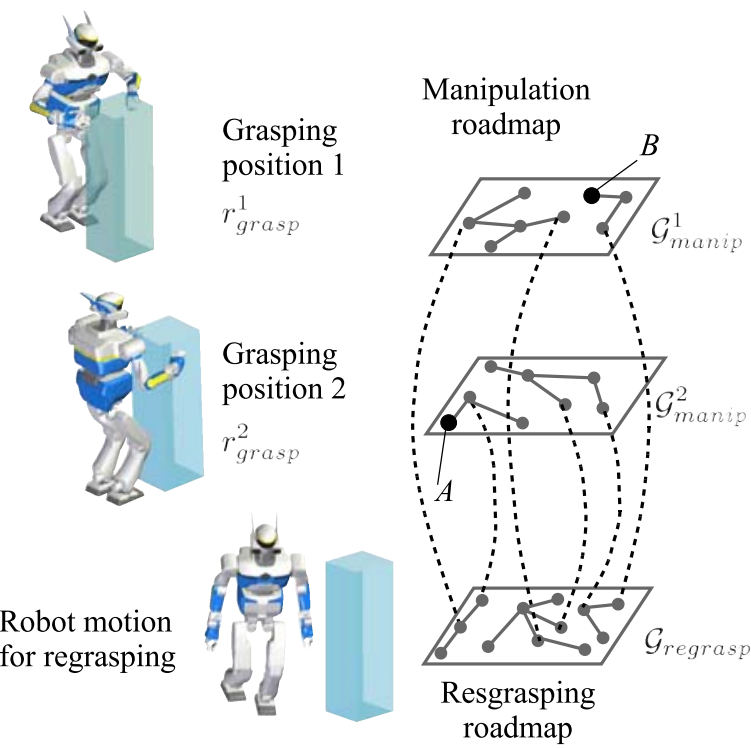

Fig. 6. Roadmap multiplexing. Different manipulation roadmaps $\mathcal{G}_{\text {manip }}^{1}$ and $\mathcal{G}_{\text {manip }}^{1}$ are connected by way of the regrasping roadmap $\mathcal{G}_{\text {reg }}$.

are two types of roadmap: the first is the "manipulation roadmap" $\mathcal{G}_{\text {manip }}^{i}$ for $i$-th grasping position $r_{\text {grasp }}^{i}$ expressed as the relative robot position with respect to object. In this roadmap, the robot and the object move together. The second type of roadmap is the "regrasping roadmap" $\mathcal{G}_{\text {reg }}$ where the robot moves alone between different grasping positions.

As can be seen in the figure, manipulation roadmaps $\mathcal{G}_{\text {manip }}^{1}$ and $\mathcal{G}_{\text {manip }}^{2}$ for different grasping positions are interconnected via the regrasping roadmap $\mathcal{G}_{\text {reg }}$. For instance, the path from $A$ to $B$ is possible only by alternating the different grasping positions.

We first use the visibility roadmap of the object $\mathcal{G}_{o b j}$ introduced in Section III to construct those roadmap. The objective of this operation is to allow the roadmaps to cover the areas around the critical configurations. Since the manipulation roadmaps generated from the object roadmap are only rough capture of the narrow passages, a fine path search is usually necessary to find the precise regrasping positions. If no solutions are found after this roadmap building operation, we adopt a diffusing roadmap exploration to grow the roadmap in narrow spaces.

The detailed procedure to research a manipulation path for the combined robot-object system is shown in Fig. 7. Since there are multiple grasping positions for the robot, the start and goal configurations are not unique. The path planning problem has then multiple start and goal sets $\mathcal{S}_{\text {start }}$ and $\mathcal{S}_{\text {goal }}$. The subroutine PlanWithObjectConfig $\left(q_{\text {obj }}, \mathcal{S}_{\text {start }}\right.$, $\mathcal{S}_{\text {goal }}$ ) makes a path search between start and goal node sets in the whole set of the roadmaps by adding a new node generated from the object configuration $q_{o b j}$.

Fig. 8 shows an example of roadmap generated from the multiplexing method. The thick line segments with black circle nodes are the roadmap built from the object roadmap and the thin line with square nodes are the result of the diffusion. We adopted a diffusion method with self parameter-tuning [22]. In this figure, roadmaps for different grasping positions 

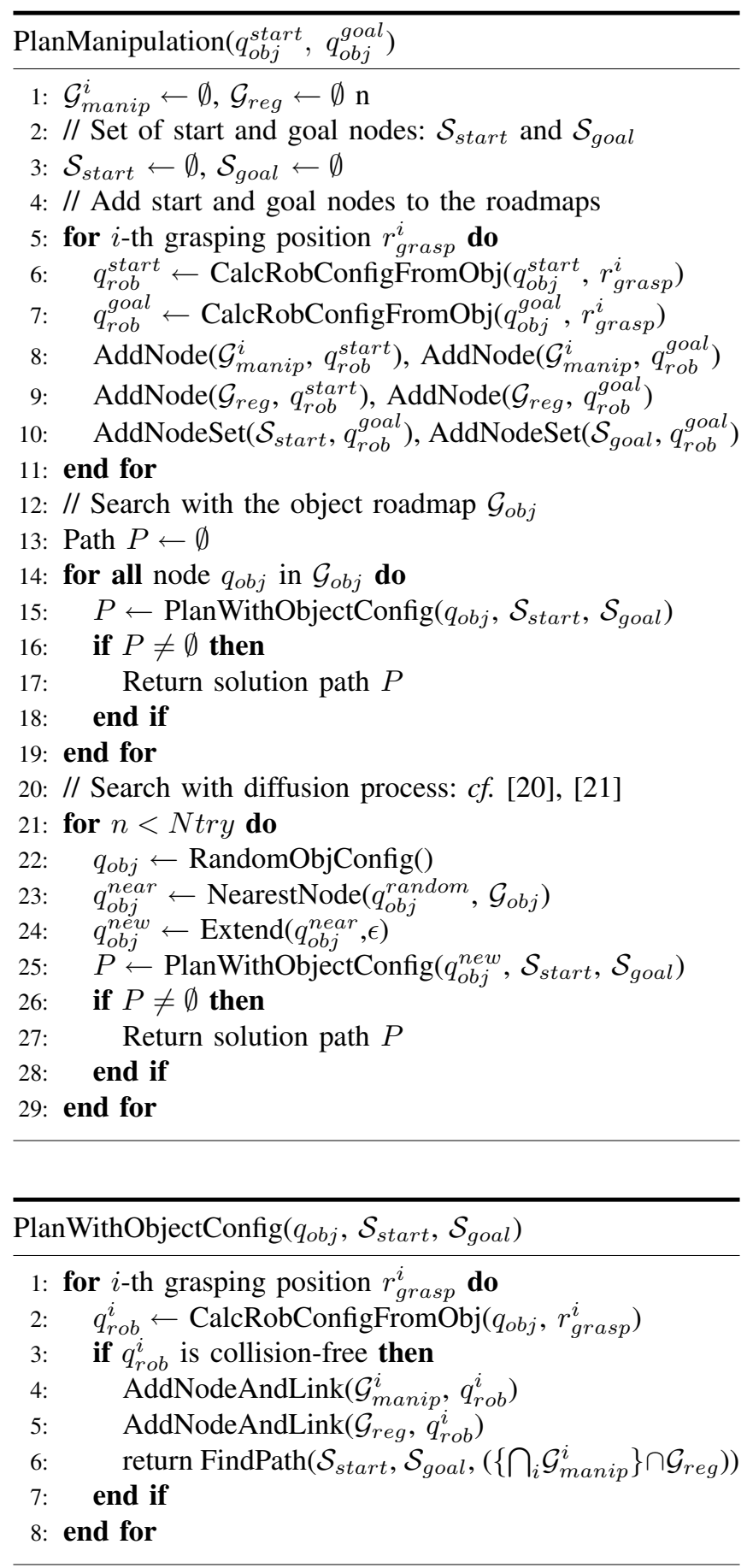

Fig. 7. Algorithm for roadmap multiplexing.

are merged. We can observe that the rough coverage from the object roadmap serves as a guide for diffusion process to find a path including regrasping.

In this framework, any local steering method that connects two configurations can be used. As mentioned earlier, we apply Reeds and Shepp curves for the object trajectory. To allow the robot to move freely to change the grasping configuration, we employ a method combining a smooth carlike robot path for forward walking and linear interpolation for sideways or backward walking in short distances.

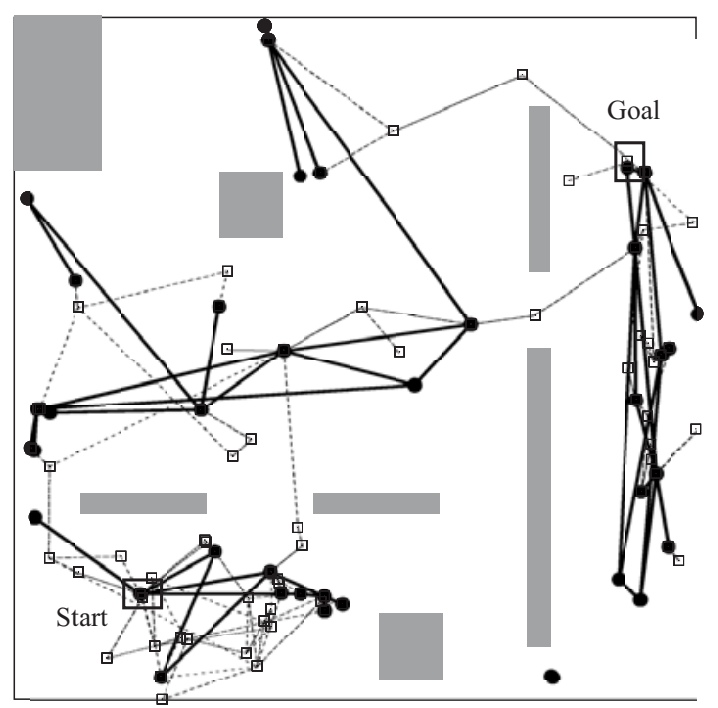

Fig. 8. Generated manipulation roadmap by the proposed method. The thick links with circle denote the roadmap derived from the object visibility-based roadmap. The thin links with squares are additional diffused roadmap.

\section{IMPLEMENTATION ISSUES}

We have implemented the proposed method in a shared software development called humanoid path planner (HPP) [23], based on a motion planning library KineoWorks ${ }^{\mathrm{TM}}$ [24]. In this framework, the software components are developed in a modular manner for the sake of reusability and easy maintenance. Thanks to the object-oriented modular architecture and common interface definitions, the developers can implement the components necessary for their own planning problem by using inherited classes of various basic libraries of motion planning.

In this research we have implemented a planning system for the proposed method of whole-body pivoting motion as shown in Fig. 9. The planner interacts with basic functions such as roadmap builders, path optimizers and collision checkers. We also implemented the steering methods specific to the pivoting planning problem.

We have developed such local steering methods as Reeds and Shepp curves for pivoting basic path, pivoting sequence generation, and path planner for free walking. We also implemented the visibility PRM builder and the multiplexed roadmap builder with combined structure of the humanoid and the object. The generalized inverse kinematics (IK) module [25] takes care of the dynamic and stable whole-body motion including the coordinated hands and legs motions.

\section{Simulation RESUlts}

The proposed method has been verified to an environment including obstacles with narrow areas. The humanoid robot HRP-2 [26] should carry a box-share object from the initial position (Fig. 10a) to its goal (Fig. 10h). First, the basic path for pivoting is planned by using the proposed method. As the resulting collision-free regrasping path is not always optimal, a path optimizer has been applied to remove redundant path components. It is then transformed to a pivoting sequence of the box and finally to the whole-body motion. 


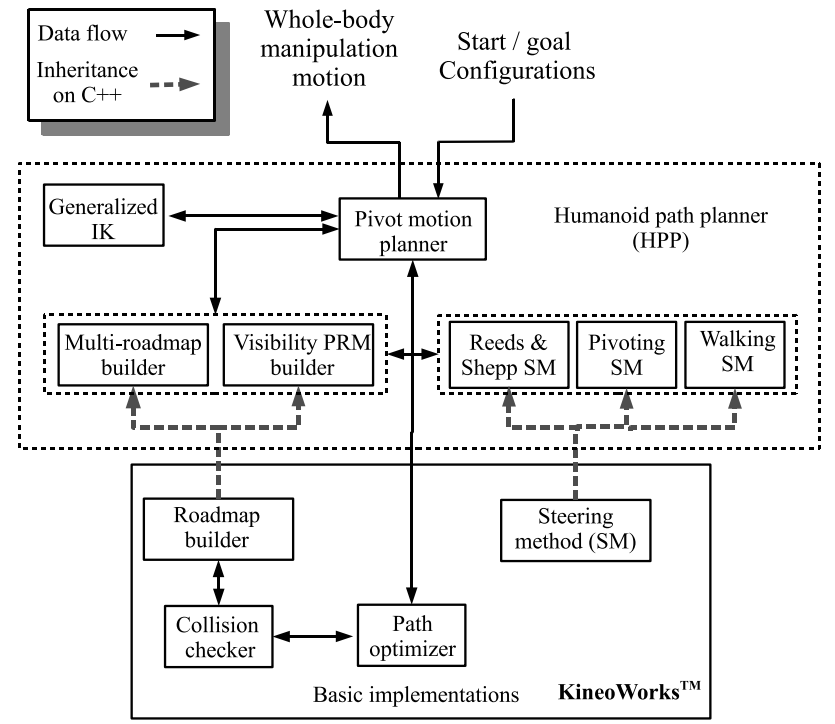

Fig. 9. Software implementation based on humanoid path planner (HPP)

The humanoid robot displaces the object at the entry of a narrow passage (Fig. 10b, c). Then it releases the object and walk to the other side of the wall (Fig. 10d). By combining backward and forward manipulation, the humanoid goes to another narrow passage (Fig. 10e, f). After another regrasping, the object is carried to the goal position (Fig. 10g, h).

The proposed method needs improvements to find a good mixture between the Visibility PRM and the diffusion algorithm. When the exploration by PRM is insufficient, the diffusion planner takes more search time. Moreover, in our example with many narrow passages, nonholonomic constraints make it difficult to connect the valid configurations. As a result, the average computation time for the example was $263.7 \mathrm{sec}$ by a PC with $2.16 \mathrm{GHz}$ Intel Centrino Duo CPU and 2GB memory. The failure ratio was $10 \%$, mainly due to the difficulty in identification of critical configurations of the object and the robot that has nonholonomic constraints.

From a theoretical point of view, the proposed multiplexing method inherits the probabilistic completeness of the PRM and the diffusion algorithms (as in [12]).

\section{CONCLUSIONS}

In this paper we have presented a method for regrasp planning for manipulation of a humanoid robot. To address this complex planning problem including manipulation and regrasping, we benefit from well-developed randomized sampling-based motion planning method. First, Visibility PRM is utilized as a preprocessing to capture the critical configurations and then roadmap multiplexing has been introduced. Manipulation roadmaps are built for each of different grasping positions whereas a regrasping roadmap interconnects them to allow the robot to change the grasping position. The set of those roadmaps are explored to find a path including regrasping that is finally transformed into a whole-body motion. A simulation result has demonstrated the validity of the proposed method.

Future work includes extension of the method to integrate different types of manipulation (lifting, pushing, etc) to reinforce the capacity of manipulation, and also improvement of the roadmap search for efficient planning. Reactive regrasp planning using sensor data to implement the proposed method with real hardware is another aspect of future development.

\section{ACKNOWLEDGMENTS}

This research was partially supported by Japan Society for the Promotion of Science (JSPS) Grant-in-Aid for Scientific Research (B), 18300070, 2006.

\section{REFERENCES}

[1] Y. Hwang, A. Konno and M. Uchiyama: "Whole body cooperative tasks and static stability evaluations for a humanoid r obot," Proc. IEEE/RSJ Int. Conf. on Intel. Robots and Syst., 1901-1906, 2003.

[2] H. Harada, S. Kajita, F. Kanehiro, K. Fujiwara, K. Kaneko, K. Yokoi, and H. Hirukawa. "Real-Time Planning of Humanoid Robot's Gait for Force Controlled Manipulation," Proc. IEEE Int. Conf. on Robotics and Automat., 616-622, 2004.

[3] H. Harada, S. Kajita, H. Saito, M. Morisawa, F. Kanehiro, K. Fujiwara, K. Kaneko , and H. Hirukawa. "A Humanoid robot carrying a heavy object," Proc. IEEE Int. Conf. on Robotics and Automat., 1712-1717, 2005.

[4] H. Arisumi, J.-R. Chardonnet, A. Kheddar, K. Yokoi, "Dynamic Lifting Motion of Humanoid Robots," Proc. IEEE Int. Conf. on Robotics and Automat., 2661-2667, 2007.

[5] Y. Aiyama, M. Inaba, and H. Inoue: "Pivoting: A new method of graspless manipulation of object by robot fingers," Proc. IEEE/RSJ Int. Conf. on Intel. Robots and Syst., 136-143, 1993.

[6] E. Yoshida, P. Blazevic, V. Hugel, K. Yokoi and K. Harada: "Pivoting a large object: whole-body manipulation by a humanoid robot," J. of Applied Bionics and Biomechanics, 3-3, 227-235, 2006.

[7] E. Yoshida, M. Poirier, J.-P. Laumond, R. Alami and K. Yokoi: "Pivoting Based Manipulation by Humanoids: a Controllability Analysis," Proc. IEEE/RSJ Int. Conf. on Intel. Robots and Syst., 1130-1135, 2007.

[8] E. Yoshida, M. Poirier, J.-P. Laumond, O. Kanoun, F. Lamiraux, R. Alami and K. Yokoi: "Whole-Body Motion Planning for Pivoting Based Manipulation by Humanoids" Proc. IEEE Int. Conf. on Robotics and Automat., 3181-3186, 2008.

[9] G. Wilfong, "Motion panning in the presence of movable obstacles," Proc. ACM Symp. Computat. Geometry, 279-288, 1988.

[10] P. Chen and Y. Hwang, "Pracitcal path planning among movable obstacles," Proc. IEEE Int. Conf. on Robotics and Automat., 444-449, 1991.

[11] J-C. Latombe, Robot Motion Planning, Kluwer Academic Publishers, 1991.

[12] T. Simeon, J-P. Laumond, J. Cortes, A. Sahbani, "Manipulation planning with probabilistic roadmaps," Int. J. Robotics Research, 23, 7-8, 729-746, 2004.

[13] M. Stilman and J. Kuffner, "Navigation among movable obstacles: Realtime reasoning in complex environments," Proc. IEEE Int. Conf. on Humanoid Robotics, 2004.

[14] M. Stilman, K. Nishiwaki, S. Kagami, and J. Kuffner, "Planning and executing navigation among movable obstacles," Proc. IEEE/RSJ Int. Conf. on Intel. Robots and Syst., 820-826, 2006.

[15] K. Okada, A. Haneda, H. Nakai, M. Inaba and H .Inoue, "Environment Manipulatio Planner for Humaonid Robots Using Task Graph That Generates Action Sequence," Proc. IEEE/RSJ Int. Conf. on Intel. Robots and Syst., 1174-1179, 2004.

[16] T. Simeon, J.-P. Laumond and C. Nissoux, "Visibility-based probabilistic roadmaps for motion planning," J. of Advanced Robotics, 14-6, 477-494, 2000.

[17] J.-P. Laumond (editor), "Robot Motion Planning and Control," Lectures Notes in Control and Information Sciences 229, Springer, 1998.

[18] J. Reeds and R. Shepp, "Optimal paths for a car that goes both forwards and backwards," Pacific J. of Mathematics, 145-2, 367-393, 1990.

[19] S. LaValle. "Planning Algorithms," Cambridge Univ. Press, 2006.

[20] D. Hsu, J.C. Latombe and R. Motwani Path Planning in Expansive Configuration Spaces Int. J. Computanional Geometry and Applications, 4, 495-512, 1999. 


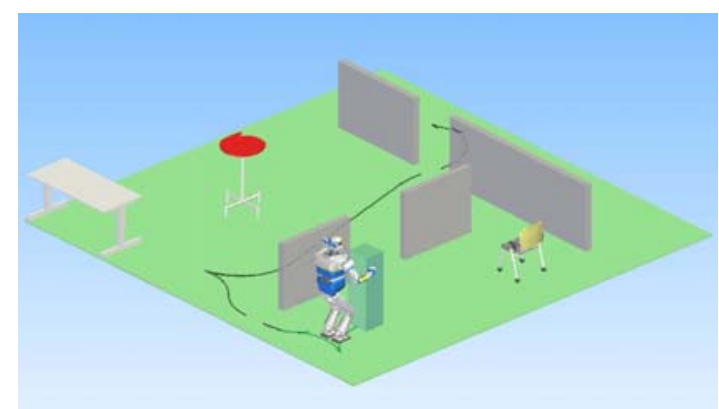

(a) Initial position

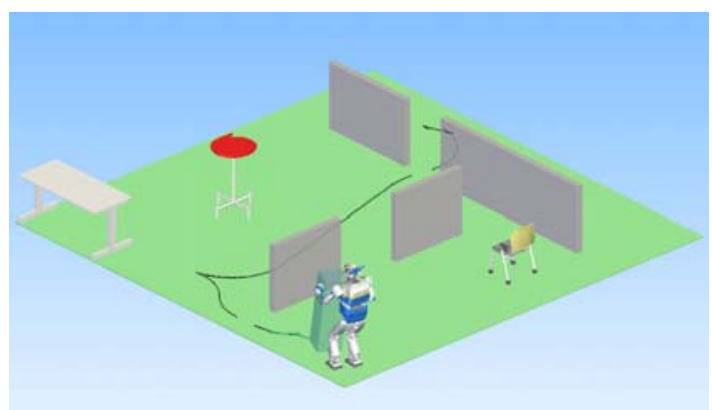

(b)

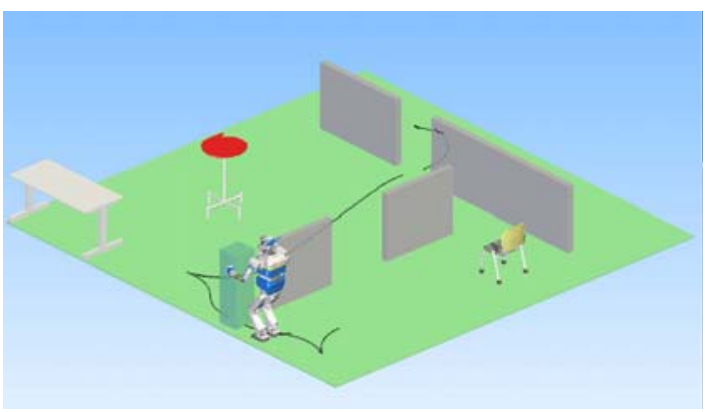

(c)

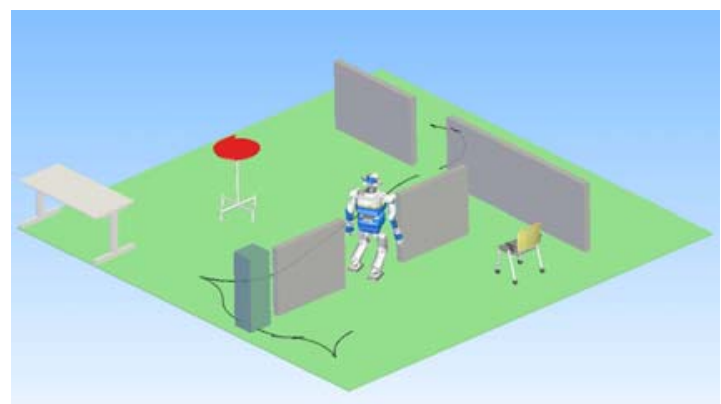

(d)

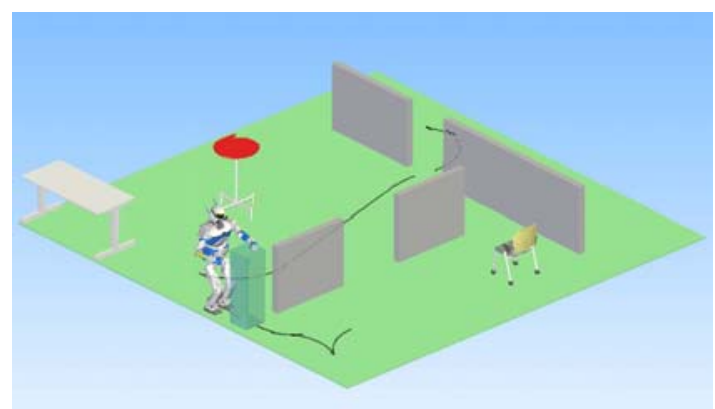

(e)

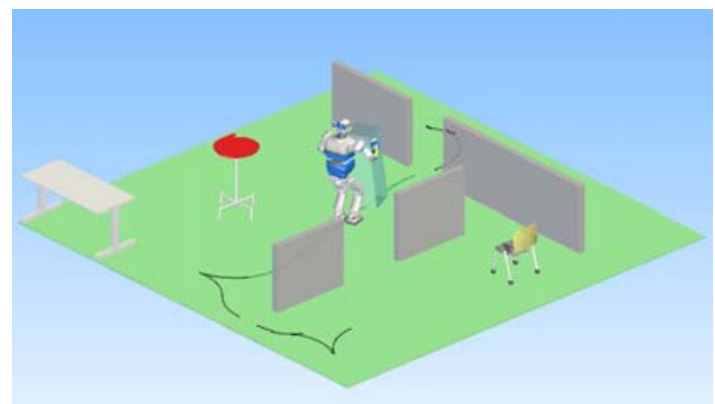

(f)

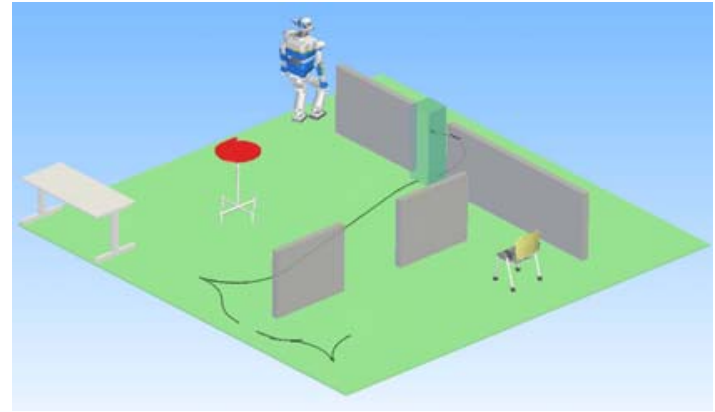

(g)

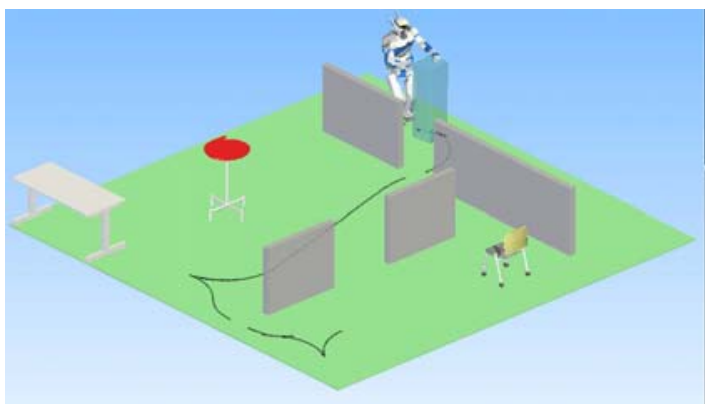

(h) Goal position

Fig. 10. Simulation result of regrasp planning. Starting from initial position (a), the humanoid robot makes pivoting sequences (b) first puts the object to the entry of passage (c). It leaves the object and walks freely by combining forward and sideways walking (d) to regrasp the object on the other side (e). Then the robot goes towards another narrow passage (f) and make another regrasp sequence (g) to arrive at the goal (h).

[21] S. LaValle and J. Kuffner, "Rapidly-Exploring Random Trees: Progress and Prospects," Algorithmic and Computational Robotics: New Directions, 293-308, A K Peters, 2001.

[22] E. Ferre and J.-P. Laumond. "An iterative diffusion algorithm for part disassembly," Proc. IEEE Int. Conf. on Robotics and Automat., 31493154, 2004.

[23] E. Yoshida, A. Mallet, F. Lamiraux, O. Kanoun, O. Stasse, M. Poirier, P. Dominey, J.-P. Laumond and K. Yokoi , "Give me the Purple Ball - he said to HRP-2 N.14," Proc. IEEE-RAS Int. Conf. on Humanoids Robots, 2007.
[24] J-P. Laumond, "Kineo CAM: a success story of motion planning algorithms," IEEE Robotics \& Automat. Magazine, 13-2, 90-93, 2006.

[25] E. Yoshida, O. Kanoun, J.-P. Laumond and C. Esteves, "Task-driven Support Polygon Reshaping for Humanoids," Proc. IEEE-RAS Int. Conf. on Humanoid Robots, 208-213, 2006.

[26] K. Kaneko, F. Kanehiro, S. Kajita, H. Hirukawa, T. Kawasaki, M. Hirata, K. Akachi and T. Isozumi, "The Humanoid Robot HRP-2", Proc. IEEE Int. Conf. on Robotics and Automat., 1083-1090, 2004. 